\title{
ASYMPTOTIC BEHAVIOUR OF CONTINUOUS TIME AND STATE BRANCHING PROCESSES
}

\author{
ZENG-HU LI
}

(Received 18 July 1997; revised 28 April 1999)

Communicated by V. Stefanov

\begin{abstract}
We prove some limit theorems for continuous time and state branching processes. The non-degenerate limit laws are obtained in critical and non-critical cases by conditioning or introducing immigration processes. The limit laws in non-critical cases are characterized in terms of the canonical measure of the cumulant semigroup. The proofs are based on estimates of the cumulant semigroup derived from the forward and backward equations, which are easier than the proofs in the classical setting.
\end{abstract}

1991 Mathematics subject classification (Amer. Math. Soc.): primary 60J80, 60F05.

Keywords and phrases: branching processes, continuous time, continuous sate, conditioning, immigration, limit theorem.

\section{Introduction}

Limit theorems constitute an important part of the theory of branching processes. Since the processes are unstable, people have derived limit theorems for them through devices such as modifying factors, conditioning, supporting immigration, and so on. A standard reference on the limit theorems for discrete state branching processes is Athreya and Ney [1]. Recently, Pakes [12] studied various conditional limit theorems for the processes in a unified setting by introducing some general conditioning events. A number of limit theorems for continuous state branching processes have also been proved in the literature; see for example [4, 16-18, 20,21].

An important feature of the continuous state branching process is that the convergence to a non-degenerate limit law can occur in supercritical, critical and subcritical

\footnotetext{
Supported by the National Natural Science Foundation of China (No. 19361060) and the JSPS

Postdoctoral Fellowship for Foreign Researchers in Japan (No. P98354).

(C) 2000 Australian Mathematical Society $0263-6115 / 2000 \$ A 2.00+0.00$
} 
cases, in contrast to the discrete state processes. Suppose that $\left\{x_{t}: t \geq 0\right\}$ is a continuous time and state branching process with $x_{0}=1$ defined on a probability space $(\Omega, \mathscr{F}, \mathrm{P})$. Let $\tau_{0}=\inf \left\{t \geq 0: x_{t}=0\right\}$ denote the extinction time of $\left\{x_{t}: t \geq 0\right\}$ and let $q_{0}=\mathbf{P}\left(\tau_{0}<\infty\right)$ be the probability of extinction. The asymptotic behavior of $\left\{x_{t}: t \geq 0\right\}$ when $q_{0}<1$ was studied by Grey [4]. Under certain hypotheses, the author showed that there are positive constants $\left\{\eta_{t}: t \geq 0\right\}$ such that $\eta_{t} x_{t}$ converges almost surely to a non-degenerate random variable as $t \rightarrow \infty$, and it holds under some moment condition that $\eta_{t} \sim c \mathrm{e}^{m t}$ for constants $c$ and $m$. These give the analogue of the classical results of Seneta and Vere-Jones [21] in the discrete time situation. In the subcritical case, the hypotheses of [4] imply that the process $\left\{x_{t}: t \geq 0\right\}$ never becomes extinct. Indeed, Pakes and Trajstman [18] showed that, under the restriction $m((0,1])<\infty$, a random time $\tau$ exists after which the process decays exponentially. That is, there exist a constant $c>0$ and a random variable $\zeta>0$ such that $x_{t}=\mathrm{e}^{-c t} \zeta$ almost surely for all $t \geq \tau$. Some limit laws of the process conditioned on $\{\tau \geq t\}$ and on $\left\{x_{t} \geq \varepsilon\right\}$ were studied in $[17,18]$; see also [2] for related work.

In this paper, we prove some conditional limit theorems for the continuous time and state branching processes which extinguish with positive probability. In the noncritical cases, we shall see a symmetry between subcritical and supercritical processes for the conditional limit theorems, which follows from the fact that a supercritical branching process conditioned on extinction is equivalent to a subcritical one. In the critical case, we consider two simplest special cases of the conditioning events of Pakes [12] and show that suitable modifications of the process lead to some universal limit laws independent of the explicit form of the branching mechanism. The analogues of those results in the discrete state situation form the core of the classical conditional limit theorems for branching processes. The continuous time and state versions are usually more complete and their proofs are more enlightening. In the non-critical cases, the conditional limit laws can be characterized in terms of the canonical measure of the cumulant semigroup. In this sense, the continuous time and state model provides a more economical way to establish the nicest conditional limit theorems for branching processes. The greater tractability of these processes arises because both their time and state space are smooth, and the distributions which appear are infinitely divisible. The proofs of the limit theorems are based on the asymptotic estimates of the cumulant semigroup derived from the forward and backward equations, which should be of interest in their own right. As an additional application of those estimates, we prove a limit theorem for the continuous time and state process with immigration, giving an analogue of the results of Foster [3] and Yamazato [22]. 


\section{Preliminaries}

Throughout this paper we consider a conservative continuous state branching process $X=\left(\Omega, \mathscr{G}, \mathscr{G}_{t}, x_{t}, Q_{x}\right)$ with branching mechanism determined by the function

$$
F(z)=-b z-c z^{2}+\int_{0}^{\infty}\left(1-\mathrm{e}^{-z u}-z u\right) m(d u), \quad z \geq 0,
$$

where $c \geq 0$ and $b$ are constants, and $\left(u \wedge u^{2}\right) m(d u)$ is a finite measure on $(0, \infty)$. For notational convenience, we define $\phi(z)=-F(z)$, which is a non-negative function when the branching mechanism is critical or subcritical. Let $\left(Q_{t}\right)_{\geq 0}$ denote the transition semigroup of $X$. Then

$$
\int_{0}^{\infty} \mathrm{e}^{-\lambda y} Q_{t}(x, d y)=\exp \left\{-x v_{t}(\lambda)\right\}, \quad \lambda \geq 0, x \geq 0,
$$

where $v_{t}(\lambda)$ is the unique positive solution to the backward equation

$$
\frac{\partial}{\partial t} v_{t}(\lambda)=-\phi\left(v_{t}(\lambda)\right), \quad v_{0}(\lambda)=\lambda, \quad t \geq 0, \lambda \geq 0 .
$$

From (2.3) we can derive the forward equation -

$$
\frac{\partial}{\partial t} v_{t}(\lambda)=-\phi(\lambda) \frac{\partial}{\partial \lambda} v_{t}(\lambda), \quad v_{0}(\lambda)=\lambda, \quad t \geq 0, \lambda \geq 0 .
$$

We may write $v_{t}^{\prime}(\lambda)$ for $(\partial / \partial \lambda) v_{t}(\lambda)$ in the sequel. Note that our moment condition on $m(d u)$ implies that $\mathbf{E}_{x} x_{t}<\infty$ for all $x \geq 0$ and $t \geq 0$. Since $v_{t}(\lambda)$ is the cumulant of an infinitely divisible non-negative random variable with finite mean, it has the canonical representation

$$
v_{t}(\lambda)=d_{t} \lambda+\int_{0}^{\infty}\left(1-\mathrm{e}^{-\lambda u}\right) l_{t}(d u), \quad t \geq 0, \lambda \geq 0,
$$

where $d_{t} \geq 0$ and $u l_{t}(d u)$ is a finite measure on $(0, \infty)$. It is well-known that the functions $\left(v_{t}\right)_{t \geq 0}$ on $[0, \infty)$ form a semigroup under composition, called the cumulant semigroup of $X$. By (2.1) and (2.3) we have $(\partial / \partial t) v_{t}(\lambda) \leq-b v_{t}(\lambda)$ and $(\partial / \partial t) v_{t}^{\prime}(0)=$ $-b v_{t}^{\prime}(0)$. It follows that $v_{t}(\lambda) \leq \mathrm{e}^{-b t} \lambda$ and $v_{t}^{\prime}(0)=\mathrm{e}^{-b t}$ for all $\lambda \geq 0$ and $t \geq 0$; see for example [1, page 259].

Let us consider the following condition on the branching mechanism:

[H] There exists $\theta>0$ such that $\phi(z)>0$ for all $z>\theta$ and $\int_{\theta}^{\infty} \phi(z)^{-1} d z<\infty$.

Clearly, under this condition $\phi(z)$ is a strictly convex function of $z \geq 0$. In the sequel, we shall write $b=\downarrow \lim _{x \uparrow a} f(x)$ to mean that $f(x)$ decreases to $b$ as $x$ increases to $a$. The arrows in $\uparrow \lim _{x \uparrow a}, \downarrow \lim _{x \downarrow a}$ and $\uparrow \lim _{x \downarrow a}$ should be interpreted in similar ways. The following result was proved in [4]. 
THEOREM 2.1 (Grey, 1974). For any $x>0$ and $t>0, \mathbf{Q}_{x}\left\{x_{t}=0\right\}>0$ if and only if $[\mathrm{H}]$ holds. Under this condition, we have

$$
\begin{aligned}
& \mathbf{Q}_{x}\left\{x_{t}=0\right\}=\exp \left\{-x \bar{v}_{t}\right\} \text { and } \\
& \mathbf{Q}_{x}\left\{x_{t}=0 \text { for some } t>0\right\}=\exp \{-x \bar{v}\}
\end{aligned}
$$

where $\bar{v}_{t}:=\uparrow \lim _{\lambda \uparrow \infty} v_{t}(\lambda)$, and $\bar{v}:=\downarrow \lim _{t \uparrow \infty} \bar{v}_{t}$ is the largest root of $\phi(z)=0$, so $\bar{v}>0$ if and only if $b=\phi^{\prime}\left(0^{+}\right)<0$.

Note that $[\mathrm{H}]$ is satisfied if $\phi(z)=c z^{1+\beta}$ for $c>0$ and $0<\beta \leq 1$, a special case excluded in [17] and [18]. In the sequel of the paper we shall always assume [H] holds. By the above theorem, we have $d_{t}=0$ and $0<l_{t}(0, \infty)=\bar{v}_{t}<\infty$ in (2.5), that is,

$$
v_{t}(\lambda)=\int_{0}^{\infty}\left(1-\mathrm{e}^{-\lambda u}\right) l_{t}(d u), \quad t>0, \lambda \geq 0 .
$$

By (2.3), for $t \geq 0$ and $\lambda>\bar{v}$ we have

$$
\int_{v_{t}(\lambda)}^{\lambda} \phi(z)^{-1} d z=t
$$

Letting $\lambda \rightarrow \infty$ in the equation (2.7) gives

$$
\int_{\bar{v}_{t}}^{\infty} \phi(z)^{-1} d z=t, \quad t>0 .
$$

Therefore, $\bar{v}_{t}=v_{t}(\infty)$ solves the equation

$$
\frac{d}{d t} \bar{v}_{t}=-\phi\left(\bar{v}_{t}\right), \quad \bar{v}_{0}=\infty, \quad t>0 .
$$

COROLLARY 2.2. For any $t>0$, the function $v_{t}(\lambda)$, for $\lambda \geq 0$ is strictly increasing and concave, and $\bar{v}$ is the largest solution to the equation $v_{t}(\lambda)=\lambda$. For $0<\lambda<\bar{v}$ we have $\bar{v}=\uparrow \lim _{t \uparrow \infty} v_{t}(\lambda)$, and for $\lambda>\bar{v}$ we have $\bar{v}=\downarrow \lim _{t \uparrow \infty} v_{t}(\lambda)$.

PROOF. By (2.6), $v_{t}(\lambda)$ is a strictly increasing and concave function for $\lambda \geq 0$. Using the semigroup property of $\left(v_{t}\right)_{t \geq 0}$ one may check that $\bar{v}_{t+r}=v_{t}\left(\bar{v}_{r}\right)$. Letting $r \rightarrow \infty$ we get $\bar{v}=v_{t}(\bar{v})$. Then $\bar{v}$ is the largest solution to the equation. If $b \geq 0$, then we have $\bar{v}=0$. If $b<0$ and $0<\lambda<\bar{v}$, then $\lambda<v_{t}(\lambda)$. Iterating this inequality we see that $v_{t}(\lambda)$ is an increasing function for $t>0$ bounded above by $\bar{v}$. Let $v_{\infty}(\lambda)=\uparrow \lim _{t \uparrow \infty} v_{t}(\lambda)$. By the relation $v_{t}\left(v_{s}(\lambda)\right)=v_{t+s}(\lambda)$ we have $v_{t}\left(v_{\infty}(\lambda)\right)=v_{\infty}(\lambda)$, and hence $v_{\infty}(\lambda)=\bar{v}$ since $\bar{v}$ is the unique solution to $v_{t}(\lambda)=\lambda$ in $(0, \infty)$. The assertion for $\lambda>\bar{v}$ can be proved similarly. 


\section{Asymptotic estimates of the cumulant semigroup}

Recall that $\lim _{t \rightarrow \infty} v_{t}(\lambda)=0$ for all $\lambda \geq 0$ in the critical and subcritical cases. In this section, we investigate the rate of this convergence, which is useful in the discussions of limit theorems. By virtue of the backward and forward equations (2.3) and (2.4) and the canonical representation (2.6), the proofs in our setting are easier and more enlightening than the classical results as developed in [1]. We start with the subcritical case.

THEOREM 3.1. Assume that $b>0$. Then for any $\lambda \geq 0$, the limit $g(\lambda):=\uparrow$ $\lim _{t \rightarrow \infty} \bar{v}_{t}^{-1} v_{t}(\lambda)$ exists and $0=g(0)=g\left(0^{+}\right) \leq g(\lambda) \leq g\left(\infty^{-}\right)=1$. Consequently, $\bar{v}_{t}^{-1} l_{t}$ converges as $t \rightarrow \infty$ to a probability measure $\pi_{0}$ on $(0, \infty)$ with Laplace transform $1-g$.

PROOF. Let $g_{t}(\lambda)=\bar{v}_{t}^{-1} v_{t}(\lambda)$ and $h_{t}(\lambda)=\lambda^{-1} v_{t}(\lambda)$. Then we have $0 \leq g_{t}(\lambda) \leq 1$ and

$$
g_{t+s}(\lambda)=v_{s}\left(\bar{v}_{t}\right)^{-1} v_{s}\left(v_{t}(\lambda)\right)=h_{s}\left(\bar{v}_{t}\right)^{-1} h_{s}\left(v_{t}(\lambda)\right) g_{t}(\lambda), \quad s \geq 0, t \geq 0 .
$$

Since $v_{t}(0)=0$ and $v_{t}(\lambda)$ is a concave function of $\lambda \geq 0$, we have $h_{t}^{\prime}(\lambda)=$ $\lambda^{-2}\left[v_{t}^{\prime}(\lambda) \lambda-v_{t}(\lambda)\right] \leq 0$, and hence $h_{t}(\lambda)$ is non-increasing in $\lambda \geq 0$. Thus $g_{t}(\lambda)$ is non-decreasing in $t \geq 0$, so the limit $g(\lambda)=\uparrow \lim _{t \rightarrow \infty} g_{t}(\lambda)$ exists and $0 \leq g(\lambda) \leq 1$ for all $\lambda \geq 0$. Since $g_{t}\left(\infty^{-}\right)=1$, we have $g\left(\infty^{-}\right)=1$. Observe that

$$
g_{t}\left(v_{s}(\lambda)\right)=g_{t+s}(\lambda) h_{s}\left(\bar{v}_{t}\right), \quad s \geq 0, t \geq 0,
$$

and $\lim _{t \rightarrow \infty} h_{s}\left(\bar{v}_{t}\right)=v_{s}^{\prime}(0)=\mathrm{e}^{-b s}$. Then letting $t \rightarrow \infty$ in (3.2) we get

$$
g\left(v_{s}(\lambda)\right)=\mathrm{e}^{-b s} g(\lambda), \quad \lambda \geq 0, s \geq 0 .
$$

It follows that $g\left(0^{+}\right)=g(0)=0$. From the relation

$$
\lim _{t \rightarrow \infty} \int_{0}^{\infty} \mathrm{e}^{-\lambda u} \bar{v}_{t}^{-1} l_{t}(d u)=1-\lim _{t \rightarrow \infty} \bar{v}_{t}^{-1} v_{t}(\lambda)=1-g(\lambda)
$$

we see that $\bar{v}_{t}^{-1} l_{t}$ converges as $t \rightarrow \infty$ to a probability measure $\pi_{0}$ on $(0, \infty)$ with Laplace transform $1-g$.

Based on (2.3) and (2.4) it is easy to check that

$$
\frac{\partial}{\partial t}\left[\log v_{t}^{\prime}(\lambda)\right]=-\phi^{\prime}\left(v_{t}(\lambda)\right)
$$


Since $v_{0}^{\prime}(\lambda)=1$, we have

$$
v_{t}^{\prime}(\lambda)=\exp \left\{-\int_{0}^{t} \phi^{\prime}\left(v_{s}(\lambda)\right) d s\right\} .
$$

Let $q_{t}(\lambda)=\mathrm{e}^{b t} v_{t}(\lambda)$ for $t \geq 0$ and $\lambda \geq 0$. By (3.5) it follows that

$$
q_{t}^{\prime}(\lambda)=\exp \left\{-\int_{0}^{t} \phi_{0}^{\prime}\left(v_{s}(\lambda)\right) d s\right\}
$$

where $\phi_{0}(z)=\phi(z)-b z$ by definition.

THEOREM 3.2. Assume that $b>0$. Then for any $\lambda \geq 0$, the limit $q^{\prime}(\lambda):=\downarrow$ $\lim _{t \rightarrow \infty} q_{t}^{\prime}(\lambda)$ exists and $0 \leq q^{\prime}(\lambda) \leq q^{\prime}(0)=1$. Moreover, $q^{\prime}(\lambda)>0$ for some (and hence all $) \lambda>0$ if and only if $\int_{1}^{\infty} u \log (u) m(d u)<\infty$. Under this condition, we have $q^{\prime}\left(0^{+}\right)=q^{\prime}(0)=1$.

PROOF. By (3.6), the first assertion follows with

$$
q^{\prime}(\lambda)=\exp \left\{-\int_{0}^{\infty} \phi_{0}^{\prime}\left(v_{s}(\lambda)\right) d s\right\} .
$$

Since $b>0$ and $v_{s}(\lambda) \leq \mathrm{e}^{-b s} \lambda$, we see easily that $q^{\prime}(\lambda)>0$ holds if and only if

$$
\int_{0}^{\infty} d s \int_{0}^{\infty} u\left(1-\exp \left\{-v_{s}(\lambda) u\right\}\right) m(d u)=\int_{0}^{\infty} u h(u, \lambda) m(d u)<\infty
$$

where

$$
h(u, \lambda):=\int_{0}^{\infty}\left(1-\exp \left\{-v_{s}(\lambda) u\right\}\right) d s
$$

Observe that

$$
\frac{\partial}{\partial u} h(0, \lambda)=\int_{0}^{\infty} v_{s}(\lambda) d s<\lambda / b,
$$

and hence $h(u, \lambda) \leq$ constant $\lambda u$ for sufficiently small $u \geq 0$. Now by our assumption on the branching mechanism we get $\int_{0}^{1} u h(u, \lambda) m(d u)<\infty$. On the other hand, by (2.3) we may change the integral variable to see that

$$
\frac{\partial}{\partial u} h(u, \lambda)=\int_{0}^{\infty} v_{s}(\lambda) \exp \left\{-v_{s}(\lambda) u\right\} d s=\int_{0}^{\lambda} v \phi(v)^{-1} \exp \{-v u\} d v .
$$

Noticing that $\phi^{\prime}(0)=b$ we have

$$
\lim _{u \rightarrow \infty} u \frac{\partial}{\partial u} h(u, \lambda)=\lim _{u \rightarrow \infty} \int_{0}^{\lambda u}(s / u) \phi(s / u)^{-1} \exp \{-s\} d s=1 / b
$$

for any $\lambda>0$. Then l'Hospital's rule implies that $\lim _{u \rightarrow \infty} h(u, \lambda) / \log u=1 / b$. Therefore, (3.8) holds if and only if $\int_{1}^{\infty} u \log (u) m(d u)<\infty$. The last assertion follows immediately by dominated convergence. 
COROLLARY 3.3. Assume that $b>0$. Then $u \mathrm{e}^{b t} l_{t}(d u)$ converges as $t \rightarrow \infty$ to a probability measure $\eta(d u)$ on $(0, \infty)$ if and only if $\int_{1}^{\infty} u \log (u) m(d u)<\infty$. Under this condition, $\eta(d u)$ has Laplace transform $q$ '.

PROOF. By the representation (2.6) we have

$$
q_{t}(\lambda)=\int_{0}^{\infty}\left(1-\mathrm{e}^{-\lambda u}\right) \mathrm{e}^{b t} l_{t}(d u),
$$

and hence

$$
q_{t}^{\prime}(\lambda)=\int_{0}^{\infty} u \mathrm{e}^{-\lambda u} \mathrm{e}^{b t} l_{t}(d u)
$$

We first regard $u \mathrm{e}^{-b t} l_{t}(d u)$ as a probability measure on $[0, \infty]$. Then Theorem 3.2 asserts that $u \mathrm{e}^{b t} l_{t}(d u)$ converges as $t \rightarrow \infty$ to a probability measure $\eta$ on $[0, \infty]$ such that

$$
q^{\prime}(\lambda)=\int_{[0, \infty]} \mathrm{e}^{-\lambda u} \eta(d u), \quad \lambda \geq 0 .
$$

Since $q_{t}^{\prime}\left(\infty^{-}\right)=0$, we have $q^{\prime}\left(\infty^{-}\right)=0$ by the monotone convergence, and hence $\eta(\{0\})=0$. Therefore, $\eta(0, \infty)=1$ holds if and only if $q_{t}^{\prime}\left(0^{+}\right)=1$, and the results follow by further applications of Theorem 3.2.

In the next theorem, we make the convention that $q_{t}(\infty)=\mathrm{e}^{b t} \bar{v}_{t}$ for $t>0$.

THEOREM 3.4. Assume that $b>0$. Then for any $0 \leq \lambda \leq \infty$, the limit $q(\lambda):=\downarrow$ $\lim _{t \rightarrow \infty} q_{t}(\lambda)$ exists. Moreover, $q(\lambda)>0$ for some (and hence all) $0<\lambda \leq \infty$ if and only if $\int_{1}^{\infty} u \log (u) m(d u)<\infty$.

PROOF. By Theorem 3.2 and dominated convergence, we have $q(\lambda)=\downarrow \lim _{t \rightarrow \infty} q_{t}(\lambda)$ for all $0 \leq \lambda \leq \infty$, where

$$
q(\lambda):=\int_{0}^{\lambda} q^{\prime}(u) d u
$$

Since $q(\infty) \geq q(\lambda)$ for all $0<\lambda<\infty$, the second assertion is immediate.

As an easy consequence of Theorems 3.1 and 3.4, we get

COROLlaRY 3.5. Assume that $b>0$. Then $\mathrm{e}^{b t} l_{t}(d u)$ converges as $t \rightarrow \infty$ to $q(\infty) \pi_{0}(d u)$, which is non-degenerate if and only if $\int_{1}^{\infty} u \log (u) m(d u)<\infty$. 
Now we consider the critical case. By virtue of the backward equation (2.3), the proof of the following result is much simpler than the one in the Galton-Watson setting given by Kesten et al [7].

THEOREM 3.6. Assume $b=0$ and $\sigma^{2}=\phi^{\prime \prime}(0)<\infty$. Then as $t \rightarrow \infty$ we have

$$
\frac{1}{t}\left[\frac{1}{v_{t}(\lambda)}-\frac{1}{\lambda}\right] \rightarrow \frac{1}{2} \sigma^{2}
$$

uniformly on $0<\lambda \leq \infty$ with the convention $1 / \infty=0$.

ProOF. For $0<\lambda \leq \infty$ and $t>0$, we may use (2.3) and (2.9) to see that

$$
\frac{1}{t}\left[\frac{1}{v_{t}(\lambda)}-\frac{1}{\lambda}\right]=-\frac{1}{t} \int_{0}^{t} \frac{1}{v_{s}(\lambda)^{2}} \frac{\partial}{\partial s} v_{s}(\lambda) d s=\frac{1}{t} \int_{0}^{t} \frac{\phi\left(v_{s}(\lambda)\right)}{v_{s}(\lambda)^{2}} d s .
$$

By l'Hospital's rule,

$$
\lim _{z \downarrow 0} \phi(z) / z^{2}=\lim _{z \downarrow 0} \phi^{\prime \prime}(z) / 2=\sigma^{2} / 2 .
$$

But by Theorem 2.1, we have $\lim _{t \rightarrow \infty} \bar{v}_{t}=0$, and hence $\lim _{t \rightarrow \infty} v_{t}(\lambda)=0$ uniformly on $0<\lambda \leq \infty$. Then the assertion follows from (3.12) and (3.13).

COROLlaRY 3.7. Assume $b=0$ and $\sigma^{2}=\phi^{\prime \prime}(0)<\infty$. Then we have

$$
\downarrow \lim _{t \rightarrow \infty} v_{t}^{\prime}(\lambda / t)=\frac{1}{\left(1+\sigma^{2} \lambda / 2\right)^{2}}, \quad \lambda \geq 0 .
$$

Proof. From (2.3) and (2.4) we have $v_{t}^{\prime}(\lambda / t)=\phi(\lambda / t)^{-1} \phi\left(v_{t}(\lambda / t)\right)$. Then (3.14) follows from Theorem 3.6.

\section{Conditional limit theorems for the non-critical case}

Let $\tau_{0}$ be the extinction time of the continuous state branching process $\left\{x_{t}: t \geq 0\right\}$. By Theorem 2.1, in the non-supercritical cases we have $\tau_{0}<\infty$ almost surely. In this section we give some conditional limit theorems of $\left\{x_{t}: t \geq 0\right\}$ based on the random time $\tau_{0}$.

We first consider a transformation of the continuous state branching process. It is easy to check that $R_{t}(x, d y):=\mathrm{e}^{b t} x^{-1} y Q_{t}(x, d y)$ defines a Markov semigroup on $(0, \infty)$. By differentiating both sides of $(2.2)$ we see that

$$
\int_{0}^{\infty} \mathrm{e}^{-\lambda y} R_{t}(x, d y)=\exp \left\{-x v_{t}(\lambda)\right\} q_{t}^{\prime}(\lambda), \quad \lambda \geq 0 .
$$


By (3.6) the Laplace transform of $\left(R_{t}\right)_{t \geq 0}$ is given by

$$
\int_{0}^{\infty} \mathrm{e}^{-\lambda y} R_{t}(x, d y)=\exp \left\{-x v_{t}(\lambda)-\int_{0}^{t} \phi_{0}^{\prime}\left(v_{s}(\lambda)\right) d s\right\}, \quad \lambda \geq 0 .
$$

Using (4.2) we may extend $\left(R_{t}\right)_{t \geq 0}$ to a transition semigroup on $[0, \infty)$, which corresponds to a special form of the continuous state branching processes with immigration studied by Kawazu and Watanabe [6], Pinsky [19], and others. In the Galton-Watson case, it was introduced by Spitzer (unpublished) and Lamperti and Ney [8]. The following theorem states that in the critical and subcritical cases $R_{t}(x, \cdot)$ is intuitively the law of $x_{t}$ conditioned on large extinction times.

THEOREM 4.1. Assume that $b \geq 0$. Then for any $x>0$ and $t \geq 0$, the distribution of $x_{t}$ under $\mathbf{Q}_{x}\left\{\cdot \mid t+r<\tau_{0}\right\}$ converges as $r \rightarrow \infty$ to $R_{t}(x, \cdot)$.

PROOF. By the Markov property we have

$$
\mathbf{Q}_{x}\left[\exp \left\{-\lambda x_{t}\right\} \mid t+r<\tau_{0}\right]=\frac{\mathbf{Q}_{r}\left[\exp \left\{-\lambda x_{t}\right\}\left(1-\exp \left\{-x_{t} \bar{v}_{r}\right\}\right)\right]}{1-\exp \left\{-x \bar{v}_{t+r}\right\}} .
$$

Recall that $\bar{v}_{t+r}=v_{t}\left(\bar{v}_{r}\right)$ and $v_{t}^{\prime}(0)=\mathrm{e}^{-b r}$. Under the hypotheses, we have $\lim _{r \rightarrow \infty} \bar{v}_{r}=0$ by Theorem 2.1. It follows that

$$
\begin{aligned}
\lim _{r \rightarrow \infty} \mathbf{Q}_{x}\left[\exp \left\{-\lambda x_{t}\right\} \mid t+r<\tau_{0}\right] & =\lim _{r \rightarrow \infty} x^{-1} \bar{v}_{r} v_{t}\left(\bar{v}_{r}\right)^{-1} \mathbf{Q}_{x}\left[x_{t} \exp \left\{-\lambda x_{t}\right\}\right] \\
& =x^{-1} \mathrm{e}^{b t} \mathbf{Q}_{x}\left[x_{t} \exp \left\{-\lambda x_{t}\right\}\right],
\end{aligned}
$$

giving the desired result.

THEOREM 4.2. Assume that $b>0$. Then $R_{t}(x, \cdot)$ converges as $t \rightarrow \infty$ to a probability measure $\eta$ on $(0, \infty)$ if and only if $\int_{1}^{\infty} u \log (u) m(d u)<\infty$. Under this condition, $\eta(d u)$ is also the limit distribution of $u \mathrm{e}^{b t} l_{t}(d u)$ described in Corollary 3.3.

Proof. Since $b>0$, we have $v_{t}(\lambda) \rightarrow 0$ as $t \rightarrow \infty$. By (4.1) and Theorem 3.2 we see that

$$
\lim _{t \rightarrow \infty} \int_{0}^{\infty} \mathrm{e}^{-\lambda y} R_{t}(x, d y)=\lim _{t \rightarrow \infty} \mathrm{e}^{b t} v_{t}^{\prime}(\lambda)=q^{\prime}(\lambda),
$$

which together with Corollary 3.3 yields the desired results.

Let $\left(Q_{t}^{\circ}\right)_{t \geq 0}$ denote the restriction to $(0, \infty)$ of the semigroup $\left(Q_{t}\right)_{t \geq 0}$. The following theorem states that $\left(Q_{t}^{\circ}\right)_{t \geq 0}$ has a $\mathrm{e}^{-b}$-invariant measure in the subcritical case. This confirms an observation of Pakes [17, page 86]. It is also closely related to the work of Pakes and Trajstman [18]. 
THEOREM 4.3. Assume that $b>0$. Then for any $x>0$ and $r \geq 0$ the distribution of $x_{t}$ under $\mathbf{Q}_{x}\left\{\cdot \mid t+r<\tau_{0}\right\}$ converges as $t \rightarrow \infty$ to a probability measure $\pi_{r}$ on $(0, \infty)$ independent of $x$. Moreover, $\pi_{0}$ is also the limit distribution of $\bar{v}_{t}^{-1} l_{t}(d u)$ and $\pi_{0} Q_{t}^{\circ}=\mathrm{e}^{-b t} \pi_{0}$ for all $t>0$.

ProOF. Taking $r=0$ in (4.3) we see that

$$
Q_{x}\left[\exp \left\{-\lambda x_{t}\right\} \mid t<\tau_{0}\right]=1-\frac{1-\exp \left\{-x v_{t}(\lambda)\right\}}{1-\exp \left\{-x \bar{v}_{t}\right\}}
$$

By Theorem 3.1 and (4.4) it follows that

$$
\lim _{t \rightarrow \infty} \mathbf{Q}_{x}\left[\exp \left\{-\lambda x_{t}\right\} \mid t<\tau_{0}\right]=1-\lim _{t \rightarrow \infty} \bar{v}_{t}^{-1} v_{t}(\lambda)=1-g(\lambda),
$$

so we get $\pi_{0}=\lim _{t \rightarrow \infty} \mathbf{Q}_{x}\left\{\cdot \mid t<\tau_{0}\right\}$. In view of (3.3) we have

$$
\int_{0}^{\infty}\left(1-\exp \left\{-v_{t}(\lambda) u\right\}\right) \pi_{0}(d u)=\int_{0}^{\infty}\left(1-\mathrm{e}^{-\lambda u}\right) \mathrm{e}^{-b t} \pi_{0}(d u),
$$

and hence $\pi_{0} Q_{t}^{\circ}=\mathrm{e}^{-b t} \pi_{0}$. By (4.3)

$$
\mathbf{Q}_{r}\left[\exp \left\{-\lambda x_{t}\right\} \mid t+r<\tau_{0}\right]=\frac{\exp \left\{-x v_{t}(\lambda)\right\}-\exp \left\{-x v_{t}\left(\lambda+\bar{v}_{r}\right)\right\}}{1-\exp \left\{-x \bar{v}_{t+r}\right\}} .
$$

As $t \rightarrow \infty$, the right hand side of (4.6) is equivalent to

$$
\bar{v}_{t+r}^{-1}\left(v_{t}\left(\lambda+\bar{v}_{r}\right)-v_{t}(\lambda)\right)=v_{t}\left(\bar{v}_{r}\right)^{-1}\left(v_{t}\left(\lambda+\bar{v}_{r}\right)-v_{t}(\lambda)\right) .
$$

Using the canonical representation we may write this into

$$
\left(\int_{0}^{\infty}\left(1-\mathrm{e}^{-\bar{v}_{r} u}\right) l_{t}(d u)\right)^{-1} \int_{0}^{\infty} \mathrm{e}^{-\lambda u}\left(1-\mathrm{e}^{-\bar{v}_{r} u}\right) l_{t}(d u),
$$

which converges as $t \rightarrow \infty$ to

$$
\left(\int_{0}^{\infty}\left(1-\mathrm{e}^{-\bar{v}_{r} u}\right) \pi_{0}(d u)\right)^{-1} \int_{0}^{\infty} \mathrm{e}^{-\lambda u}\left(1-\mathrm{e}^{-\bar{v}_{r} u}\right) \pi_{0}(d u),
$$

giving the Laplace transform of a probability $\pi_{r}$ on $(0, \infty)$.

COROLLARY 4.4. Assume that $b>0$ and let $\pi_{0}$ be given by Theorem 4.3. Then we have $\int_{0}^{\infty} u \pi_{0}(d u)=q(\infty)^{-1}$. Thus $\pi_{0}$ has finite mean if and only if $\int_{1}^{\infty} u \log (u) m(d u)$ $<\infty$. 
PrOOF. By the proof of Theorem 4.3, the measure $\pi_{0}$ has Laplace transform 1 $g(\lambda)$. Letting $\lambda \rightarrow \infty$ in (3.3) we have $g\left(\bar{v}_{s}\right)=\mathrm{e}^{-b s}$. It follows that

$$
\int_{0}^{\infty} u \pi_{0}(d u)=g^{\prime}(0)=\lim _{s \rightarrow \infty} \bar{v}_{s}^{-1} \mathrm{e}^{-b s}=\lim _{s \rightarrow \infty} q_{s}(\infty)^{-1}=q(\infty)^{-1},
$$

which together with Theorem 3.4 gives the desired conclusion.

The above conditional limit theorems are not restricted to the subcritical cases. In fact, there is a symmetry in the limit theorems between the subcritical and supercritical processes if we use suitable conditioning. In the supercritical case, we have $b<0$ and

$$
\mathbf{Q}_{x}\left[\exp \left\{-\lambda x_{t}\right\} \mid \tau_{0}<\infty\right]=\exp \left\{-x w_{t}(\lambda)\right\}, \quad \lambda \geq 0,
$$

where $w_{t}(\lambda)=v_{t}(\lambda+\bar{v})-\bar{v}$. Setting $\phi_{\bar{v}}(\lambda)=\phi(\lambda+\bar{v})$ one may see that $w_{t}(\lambda)$ satisfies

$$
\frac{\partial}{\partial t} w_{t}(\lambda)=-\phi_{i}\left(w_{t}(\lambda)\right), \quad w_{0}(\lambda)=\lambda, \quad t \geq 0, \lambda \geq 0 .
$$

It is not hard to check that $-\phi_{\bar{v}}(\lambda):=\phi(\bar{v})-\phi(\lambda+\bar{v})$ has the representation (2.1) with parameters $b_{\bar{v}}:=\phi^{\prime}(\bar{v}), c_{\bar{v}}:=c$ and $m_{\bar{v}}(d u):=\mathrm{e}^{-\bar{v} u} m(d u)$. Recall that $\bar{v}>0$ is the largest root of $\phi(z)=z$, so we have $b_{\bar{v}}>0$. Therefore, (4.8) implies that $\left\{x_{t}: t \geq 0\right\}$ conditioned on $\tau_{0}<\infty$ is a subcritical continuous state branching process with cumulant semigroup $\left(w_{t}\right)_{t \geq 0}$. In particular, $w_{t}(\lambda)$ has the representation $(2.6)$ with canonical measure $\mathrm{e}^{-\bar{v} u} l_{t}(d u)$. Using Theorem 4.1, Theorem 4.2 and Theorem 4.3 we get the following

THEOREM 4.5. Assume that $b<0$. Then for any $x>0$ the distribution of $x_{t}$ under $\mathbf{Q}_{x}\left\{\cdot \mid t+r<\tau_{0}<\infty\right\}$ converges as $r \rightarrow \infty$ to a probability measure $T_{t}(x, \cdot)$ on $(0, \infty)$. Moreover, $T_{t}(x, \cdot)$ converges as $t \rightarrow \infty$ to a probability measure $\eta(d u)$ on $(0, \infty)$, which is also the limit distribution of $u \mathrm{e}^{b_{\hat{v}} t} \mathrm{e}^{-\bar{v} u} l_{t}(d u)$.

THEOREM 4.6. Assume that $b<0$. Then for $x>0$ and $r \geq 0$ the distribution of $x_{t}$ under $\mathbf{Q}_{x}\left\{\cdot \mid t+r<\tau_{0}<\infty\right\}$ converges as $t \rightarrow \infty$ to a probability measure $\pi_{r}$ on $(0, \infty)$ which is independent of $x$. Moreover, $\pi_{0}(d u)$ is also the limit distribution of $\left(\bar{v}_{t}-\bar{v}\right)^{-1} \mathrm{e}^{-\bar{v} u} l_{t}(d u)$.

\section{Conditional limit theorems for the critical case}

Now we consider the critical case. The following theorems show that suitable conditioning of the critical process may lead to some universal limit laws independent of the explicit form of the branching mechanism. We first give an analogue of the result of Harris [5]. 
THEOREM 5.1. Assume that $b=0$ and $\sigma^{2}=\phi^{\prime \prime}(0)<\infty$. Let $\left\{y_{t}: t \geq 0\right\}$ be a Markov process with transition semigroup $\left(R_{t}\right)_{t \geq 0}$. Then the distribution of $y_{t} / t$ converges as $t \rightarrow \infty$ to the one on $[0, \infty)$ with density $4 x \exp \left\{-2 x / \sigma^{2}\right\} / \sigma^{4}$.

ProOF. Since $b=0$, we have $q_{t}^{\prime}(\lambda)=v_{t}^{\prime}(\lambda)$ by (2.6) and (3.10). By (4.1) and Corollary 3.7 we see that

$$
\lim _{t \rightarrow \infty} \int_{0}^{\infty} \mathrm{e}^{-\lambda y / t} R_{t}(x, d y)=\lim _{t \rightarrow \infty} q_{t}^{\prime}(\lambda / t)=\left(1+\sigma^{2} \lambda / 2\right)^{-2},
$$

which is the Laplace transform of the desired limit distribution.

A number of conditional limit theorems for Galton-Watson processes were proved in [12] by introducing some general conditioning events, which unify the known results. The following theorems treat two simplest special cases of those conditional events. The results will be used in the next section to draw a limit theorem for the branching process with state dependent immigration. We shall give the sketches of their proofs, for they are simpler than those of [12] in the more sophisticated situation.

THEOREM 5.2. Assume that $b=0$ and $\sigma^{2}=\phi^{\prime \prime}(0)<\infty$. Then for any fixed $x>0$ and $r \geq 0$,

$$
\lim _{t \rightarrow \infty} Q_{x}\left\{x_{t} / t>z \mid t+r_{-} \leqslant \tau_{0}\right\}=\exp \left\{-2 z / \sigma^{2}\right\}, \quad z \geq 0 .
$$

Proof. By (4.3) we have

$$
\mathbf{Q}_{x}\left[\exp \left\{-\lambda x_{t} / t\right\} \mid t+r<\tau_{0}\right]=\frac{\exp \left\{-x v_{t}(\lambda / t)\right\}-\exp \left\{-x v_{t}\left(\lambda / t+\bar{v}_{r}\right)\right\}}{1-\exp \left\{-x \bar{v}_{t+r}\right\}}
$$

As $t \rightarrow \infty$, the right hand side is equivalent to

$$
\left(v_{t}\left(\lambda / t+\bar{v}_{r}\right)-v_{t}(\lambda / t)\right) / \bar{v}_{t+r} .
$$

By Theorem 3.6 we have $\lim _{t \rightarrow \infty} t \bar{v}_{t+r}=2 / \sigma^{2}$ and $\lim _{t \rightarrow \infty} t v_{t}(\lambda / t)=\left(1 / \lambda+\sigma^{2} / 2\right)^{-1}$. From the uniform convergence we get

$$
\sigma^{2} / 2=\lim _{t \rightarrow \infty} \frac{1}{t}\left[\frac{1}{v_{t}\left(\lambda / t+\bar{v}_{r}\right)}-\frac{1}{\lambda / t+\bar{v}_{r}}\right]=\lim _{t \rightarrow \infty}\left[t v_{t}\left(\lambda / t+\bar{v}_{r}\right)\right]^{-1} .
$$

Then it follows that

$$
\lim _{t \rightarrow \infty} \mathbf{Q}_{x}\left[\exp \left\{-\lambda x_{t} / t\right\} \mid t+r<\tau_{0}\right]=\left(1+\sigma^{2} \lambda / 2\right)^{-1},
$$

yielding the desired result. 
THEOREM 5.3. Assume that $b=0$ and $\sigma^{2}=\phi^{\prime \prime}(0)<\infty$. Then for any $x>0$ and $\alpha \geq 0$ the distribution of $x_{t} / t$ under $\mathbf{Q}_{x}\left\{\cdot \mid(1+\alpha) t<\tau_{0}\right\}$ converges as $t \rightarrow \infty$ to the one on $[0, \infty)$ with density

$$
2(1+\alpha) \exp \left\{-2 x / \sigma^{2}\right\}\left[1-\exp \left\{-2 x / \sigma^{2} \alpha\right\}\right] / \sigma^{2} .
$$

PROOF. Taking $r=\alpha t$ in (4.2) we get

$$
\mathbf{Q}_{x}\left[\exp \left\{-\lambda x_{t} / t\right\} \mid(1+\alpha) t<\tau_{0}\right]=\frac{\exp \left\{-x v_{t}(\lambda / t)\right\}-\exp \left\{-x v_{t}\left(\lambda / t+\bar{v}_{\alpha t}\right)\right\}}{1-\exp \left\{-x \bar{v}_{(1+\alpha) t}\right\}},
$$

which is equivalent to

$$
\left(v_{t}\left(\lambda / t+\bar{v}_{\alpha t}\right)-v_{t}(\lambda / t)\right) / \bar{v}_{(1+\alpha) t}
$$

as $t \rightarrow \infty$. Using Theorem 3.6 one can show that

$$
\lim _{t \rightarrow \infty} \mathbf{Q}_{x}\left[\exp \left\{-\lambda x_{t} / t\right\} \mid(1+\alpha) t<\tau_{0}\right]=\frac{1+\alpha}{\left(1+\sigma^{2} \lambda / 2\right)\left(1+\alpha+\alpha \sigma^{2} \lambda / 2\right)}
$$

which is the Laplace transform of the distribution with density (5.3).

\section{A limit theorem for the immigration process}

In this section, we consider a modification of the continuous state branching process, which allows immigration after the population becomes extinct. Its discrete state analogues have been studied by Foster [3], Pakes [13-15], Mitov and Yanev [10,11], Yamazato [22] and many others. Suppose that $q>0$ is a constant and $G$ is a probability measure on $(0, \infty)$ satisfying

$$
g:=\int_{0}^{\infty} y G(d y)<\infty .
$$

Let $\left\{\tau^{i}: i=0,1,2, \ldots\right\}$ be a family of independent identically distributed exponential random variables with parameter $q>0$ and let $\left\{x^{i}(t): t \geq 0 ; i=1,2, \ldots\right\}$ be a family of independent identically distributed continuous state branching processes with initial distribution $G$. We define another continuous state branching process $\left\{x^{0}(t): t \geq 0\right\}$ with the same transition semigroup as $\left\{x^{i}(t): t \geq 0 ; i=1,2, \ldots\right\}$, but its initial distribution is not specified. Suppose that the families $\left\{\tau^{i}: i=0,1,2, \ldots\right\}$, $\left\{x^{i}(t): t \geq 0 ; i=1,2, \ldots\right\}$ and $\left\{x^{0}(t): t \geq 0\right\}$ are independent of each other. Let $\tau_{0}^{i}:=\inf \left\{t \geq 0: x^{i}(t)=0\right\}$ denote the extinction time of $\left\{x^{i}(t): t \geq 0\right\}$. Let

$$
\sigma_{n}:=\sum_{i=0}^{n}\left[\tau_{0}^{i}+\tau^{i}\right]
$$


Then $\sigma_{n} \rightarrow \infty$ almost surely as $n \rightarrow \infty$. We define the process $\left\{y_{t}: t \geq 0\right\}$ by

$$
y_{t}= \begin{cases}x^{0}(t) & \text { for } 0 \leq t<\tau_{0}^{0}+\tau^{0}, \\ x^{n}\left(t-\sigma_{n-1}\right) & \text { for } \sigma_{n-1} \leq t<\sigma_{n} \text { and } n \geq 1 .\end{cases}
$$

It is not hard to check that $\left\{y_{t}: t \geq 0\right\}$ is a time homogeneous Markov process, which will be called an immigration process with waiting parameter $q$ and returning distribution $G$. Note that the immigration process started with zero is a special case of the regenerative process of Mitov et al [9], which was constructed by restarting independent copies of a general stochastic process after the hitting times to zero.

The transition semigroup $\left(R_{t}\right)_{t \geq 0}$ of $\left\{y_{t}: t \geq 0\right\}$ can be computed based on the construction (6.3). For any $x>0$ the increasing function $Q_{t}(x,\{0\})$ of $t \geq 0$ determines a probability measure $Q(d t, x,\{0\})$ on $(0, \infty]$ with density $x \phi\left(\bar{v}_{t}\right) \exp \left\{-x \bar{v}_{t}\right\}$. Then

$$
h(t)=\int_{0}^{t} q \mathrm{e}^{-q s} d s \int_{0}^{\infty} x \exp \left\{-x \bar{v}_{t-s}\right\} \phi\left(\bar{v}_{t-s}\right) G(d x), \quad t \geq 0
$$

defines the density of a probability measure $H(d t)$ on $[0, \infty)$. Indeed, $H(d t)$ gives the distribution of the random variables $\left\{\tau_{0}^{i}+\tau^{i}: i=1,2, \ldots\right\}$. We define the renewal measure $U(d t)$ on $[0, \infty)$ by

$$
U(d t)=\sum_{n=0}^{\infty} H^{* n}(d t), \quad t \geq 0,
$$

where $H^{* n}$ denotes the $n$-fold convolution of $H$ with $H^{* 0}=\delta_{0}$ by convention. Set $H(t)=H([0, t])$ and $U(t)=U([0, t])$. Observe that for any $t \geq 0$ we have

$$
U(t) \leq \sum_{n=0}^{\infty} H(t)^{n}=\frac{1}{1-H(t)} .
$$

A characterization for the transition semigroup of the immigration process $\left\{y_{t}: t \geq 0\right\}$ is given as follows.

THEOREM 6.1. For any bounded Borel function $f$ on $[0, \infty)$ we have

$$
R_{t} f(0)=f(0)-\int_{0}^{t} U(d s) \int_{0}^{t-s} q \mathrm{e}^{-q r} d r \int_{0}^{\infty}\left[f(0)-Q_{t-s-r} f(y)\right] G(d y)
$$

and

$$
R_{t} f(x)=Q_{t} f(x)-\int_{0}^{t}\left[f(0)-R_{t-s} f(0)\right] Q(d s, x,\{0\}), \quad x>0 .
$$


Proof. In the sequel we may write $G(f)$ for $\int_{0}^{\infty} f(y) G(d y)$. Since $\sigma_{n} \rightarrow \infty$ almost surely as $n \rightarrow \infty$, for any $t \geq 0$ we have

$$
\mathbf{P}\left(0 \leq t \leq \sigma_{0} \text { or } \sigma_{n-1}<t \leq \sigma_{n} \text { for some } n \geq 1\right)=1 .
$$

If $f(0)=0$, an easy computation based on (6.3) leads to

$$
R_{\imath} f(0)=\int_{0}^{t} U(d s) \int_{0}^{t-s} q \mathrm{e}^{-q r} G\left(Q_{t-s-r} f\right) d r
$$

Similarly we have

$$
R_{t}(0,\{0\})=\int_{0}^{t} \mathrm{e}^{q(s-t)} U(d s)
$$

For a general bounded Borel function $f$ on $[0, \infty)$ we can combine the above to get

$$
R_{t} f(0)=\int_{0}^{t} U(d s) \int_{0}^{t-s} q \mathrm{e}^{-q r} G\left(Q_{t-s-r}^{\circ} f\right) d r+\int_{0}^{t} \mathrm{e}^{q(s-t)} U(d s) f(0) .
$$

Using this and the renewal equation for $U(d t)$ we can compute that

$$
\begin{aligned}
R_{t} f(0)= & \int_{0}^{t} U(d s) \int_{0}^{t-s} q \mathrm{e}^{-q r} G\left(Q_{t-s-r} f\right) d r \\
& -\int_{0}^{t} H(t-s) U(d s) f(0)+\int_{0}^{t} \mathrm{e}^{q(s-t)} U(d s) f(0) \\
= & \int_{0}^{t} U(d s) \int_{0}^{t-s} q \mathrm{e}^{-q r}\left[G\left(Q_{t-s-r} f\right)-f(0)\right] d r \\
& +\int_{0}^{t}[1-H(t-s)] U(d s) f(0)
\end{aligned}
$$

By the strong Markov property of the process $\left\{y_{t}: t \geq 0\right\}$,

$$
R_{t} f(x)=Q_{t}^{\circ} f(x)+\int_{0}^{t} R_{t-s} f(0) Q(d s, x,\{0\}), \quad x>0 .
$$

Then (6.8) is immediate.

LEMMA 6.1. Assume $b=0$ and $\sigma^{2}=\phi^{\prime \prime}(0)<\infty$. Then, as $t \rightarrow \infty$,

$$
t^{2} h(t) \rightarrow 2 g / \sigma^{2} \text { and } t[1-H(t)] \rightarrow 2 g / \sigma^{2}
$$

where $g$ is defined by (6.1). 
PROOF. We first rewrite (6.4) as

$$
h(t)=\int_{0}^{\infty} q \mathrm{e}^{-q s} 1_{\{s \leq t)} d s \int_{0}^{\infty} x \exp \left\{-x \bar{v}_{t-s}\right\} \phi\left(\bar{v}_{t-s}\right) G(d x), \quad t \geq 0 .
$$

By Theorem 3.6 and (3.13) we have $t^{2} \phi\left(\bar{v}_{t-s}\right) \rightarrow 2 / \sigma^{2}$ as $t \rightarrow \infty$. Then the results follow by dominated convergence theorem and the l'Hospital's rule.

Now we present a limit theorem for the immigration process. The following result gives a continuous time and space version of the theorems of Foster [3] and Yamazato [22].

THEOREM 6.2. Assume that $b=0$ and $\sigma^{2}=\phi^{\prime \prime}(0)<\infty$. Let $Y=\left(\Omega, \mathscr{G}, \mathscr{G}_{t}, y_{t}\right.$, $\mathbf{R}_{x}$ ) be a realization of the semigroup $\left(R_{t}\right)_{t \geq 0}$. Then for any $x \geq 0$ and $0<\beta<1$ we have

$$
\lim _{t \rightarrow \infty} \mathbf{R}_{x}\left\{\log y_{t} / \log t \leq \beta\right\}=\beta .
$$

PROOF. Under $\mathbf{R}_{0}$, the process $\left\{y_{t}: t \geq 0\right\}$ has the same law as the one constructed by (6.3) with $x_{t}^{0}=0$ for all $t \geq 0$, which is a special form of the regenerative process studied by Mitov et al [9]. With the results in the previous sections, it is not hard to check that all the conditions in Theorem 2 of [9] are satisfied. Consequently, we have

$$
\lim _{t \rightarrow \infty} \mathbf{R}_{0}\left\{\delta\left(y_{t}\right) / \delta(t) \leq \beta\right\}=\beta,
$$

where $\delta(t):=\int_{0}^{t}(1-H(s)) d s$. By Lemma 6.1 we see that $(6.11)$ holds for $x=0$. Then the desired result follows by (6.8).

\section{Acknowledgments}

I thank Professors A. G. Pakes and N. M. Yanev for sending me their papers, on which the work is based. I am extremely grateful to the referee whose suggestions have led to significant improvements of the paper. A number of corrections have also been suggested by $X$. M. Fan.

\section{References}

[1] K. B. Athreya and P. E. Ney, Branching processes (Springer, Berlin, 1972).

[2] N. H. Bingham, 'Continuous branching processes and spectral positivity', Stochastic Process. Appl. 4 (1976), 217-242. 
[3] J. H. Foster, 'A limit theorem for a branching process with state-dependent immigration', Ann. Math. Statist. 42 (1971), 1773-1776.

[4] D. R. Grey, 'Asymptotic behavior of continuous time, continuous state-space branching processes', J. Appl. Probab. 11 (1974), 669-677.

[5] T. E. Harris, 'Some mathematical models for branching processes', in: Second Symposium on Probability and Statistics (ed. J. Neyman) (University of California Press, 1951) pp. 305-328.

[6] K. Kawazu and S. Watanabe, 'Branching processes with immigration and related limit theorems', Theory Probab. Appl. 16 (1971), 36-54.

[7] H. Kesten, P. Ney and F. Spitzer, 'The Galton-Watson process with mean one and finite variance', Theory Probab. Appl. 11 (1966), 513-540.

[8] J. Lamperti and P. Ney, 'Conditioned branching processes and their limiting diffusions', Theory Probab. Appl. 13 (1968), 128-139.

[9] K. V. Mitov, S. A. Grishechkin and N. M. Yanev, 'Limit theorems for regenerative processes', $C$. R. Acad. Bulgare Sci. 49 (1996), 29-32.

[10] K. V. Mitov and N. M. Yanev, 'Bellman-Harris branching processes with state-dependent immigration', J. Appl. Probab. 22 (1985), 757-765.

[11] _-, 'Bellman-Harris branching processes with a special type of state-dependent immigration', Adv. Appl. Probab. 21 (1989), 270-283.

[12] A. G. Pakes, 'Revisiting conditional limit theorems for mortal simple branching processes', Bernoulli (to appear).

[13] - 'A branching process with a state dependent immigration component', Adv. Appl. Probab. 3 (1971), 301-314.

[14] — - 'Some results on non-supercritical Galton-Watson processes with immigration', Math. Biosci. 24 (1975), 71-92.

[15] - 'On the age distribution of a Markov chain', J. Appl. Probab. 15 (1978), 65-77.

[16] —-, 'Some limit theorems for Jiřina processes', Period. Math. Hung. 10 (1979), 55-66.

[17] —, 'Some limit theorems for continuous-state branching processes', J. Austral. Math. Soc. (Ser. A) 44 (1988), 71-87.

[18] A. G. Pakes and A. C. Trajstman, 'Some properties of continuous-state branching processes, with applications to Bartoszynski's virus model', Adv. Appl. Probab. 17 (1985), 23-41.

[19] M. A. Pinsky, 'Limit theorems for continuous state branching processes with immigration', Bull. Amer. Math. Soc. 78 (1972), 242-244.

[20] E. Seneta and D. Vere-Jones, 'On the asymptotic behavior of branching processes with continuous state space', Z. Wahrsch. Verw. Gebiete 10 (1968), 212-225.

[21] —_, 'On a problem of Jiřina concerning continuous state branching processes', Czechoslovak Math. J. 19 (1969), 277-283.

[22] M. Yamazato, 'Some results on continuous time branching processes with state-dependent immigration', J. Math. Soc. Japan 27 (1975), 479-496.

\section{Department of Mathematics}

Beijing Normal University

Beijing 100875

P. R. China

e-mail: lizh@email.bnu.edu.cn 